\title{
Simulation of Main Steam Temperature Control System Based on Neural Network
}

\author{
Fengzhi Dai, Yujie Yan, Baochang Wei, Yuxing Ouyang, Lingran An \\ College of Electronic Information and Automation, \\ Tianjin University of Science and Technology, China; \\ E-mail:daifz@tust.edu.cn \\ www.tust.edu.cn
}

\begin{abstract}
This paper designs a PID control system based on the BP neural network. The control system can adjust three adjustable parameters of PID controller through BP neural network algorithm. The main steam temperature control system of the thermal power plant was taken as the object, and simulation analysis was performed through MATLAB. The experimental results show that the design scheme has good performance and anti-interference ability, and has certain practicability to the main steam temperature control system of thermal power plant.
\end{abstract}

Keywords: BP neural network, PID control, MATLAB, temperature

\section{Introduction}

Control theory has undergone several stages of development and made historic contributions to aerospace, military and industry. However, both the classical control theory and the modern control theory rely too much on the mathematical model, which limits these applications greatly ${ }^{1}$.

Main plant temperature control system is a typical large delay, nonlinear and time-varying system ${ }^{2}$. If we use a conventional PID controller, we will not achieve the desired control effect. The work of this paper is to select the most widely used BP neural network algorithm and to control the main steam temperature of the power plant by combining the control strategy of three-layer BP neural network with PID classic incremental numerical control algorithm. Because the main steam temperature of the power plant is a time-varying uncertain object, it has different mathematical models under different load values. Basing on the data of the literature ${ }^{3}$, we conducted simulations under different loads. In addition to verifying the following performance of the neural network PID control system, it also increases the test of anti-jamming capability. It is another practical attempt and exploration to combine the traditional PID control and neural network to control to achieve the main steam temperature control ${ }^{4}$.

\section{PID temperature control system based on BP neural network}

BP neural network is a kind of multi-layer feed-forward network. It follows the error backwards and can learn a lot of input and output information. To minimize the error of network, it uses the steepest descent learning method to adjust the threshold and weight values by the back propagation.

\section{2-1. PID control system based on neural network}

In the actual control system, the PID controller still occupy more than $90 \%$. In this paper, the advantages of the classical PID controller and neural network will be shown. As long as the input-output error of the system is not 0 , the system continuously adjusts the neural network weights to obtained optimal PID controller parameters. 
PID control system based on neural network is shown in Fig.1.

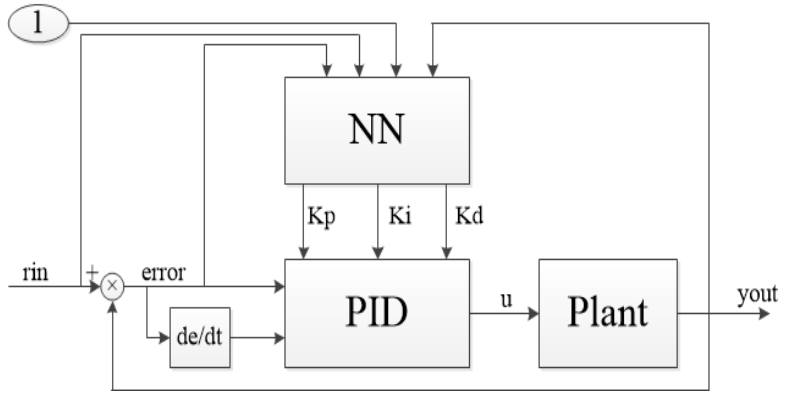

Fig.1 PID control system based on neural network

\section{2-2. Algorithm of PID control system based on BP neural network}

The expression of the incremental PID control algorithm is:

$$
\begin{aligned}
& u(k)=u(k-1)+k_{p}(e(k)-e(k-1))+k_{i} e(k) \\
& +k_{d}(e(k)-2 e(k-1)+e(k-2))
\end{aligned}
$$

Because the $k_{p}, k_{i}, k_{d}$ are adjustable coefficients, the above formula can be expressed as:

$$
\begin{aligned}
& u(k)=f\left[u(k-1), k_{p}, k_{i}, k_{d}, e(k), e(k-1),\right. \\
& e(k-2)]
\end{aligned}
$$

Among them, $f\left(^{*}\right)$ is a nonlinear function trained by neural network. This design chooses 4-5-3 BP neural network structure. Because the three coefficients must be positive, the activation function of the neurons in the output layer is a non-negative ${ }^{5}$. The formula is as follows:

$$
g(x)=\frac{1}{2}(1+\tanh (x))=\frac{e^{x}}{e^{x}+e^{-x}}
$$

The activation function of hidden layer neurons is:

$$
f(x)=\tanh (x)=\frac{e^{x}+e^{-x}}{e^{x}+e^{-x}}
$$

Select the performance index function $\mathrm{E}(\mathrm{k})$, our goal is to minimize $\mathrm{E}(\mathrm{k})$ :

$$
E(k)=\frac{1}{2}(\operatorname{rin}(k)-\operatorname{yout}(k))^{2}
$$

Use gradient descent method to correct neural network weight coefficient:

$$
\Delta w_{l i}^{(3)}(k)=-\eta \frac{\partial E(k)}{\partial w_{l i}^{(3)}}+\alpha \Delta w_{l i}^{(3)}(k-1)
$$

In the formula, $\alpha$ is the inertia coefficient and $\eta$ is the learning rate. The above formula adds the inertia term to make the system converge quickly. Through calculation and simplification, get the output layer weight calculation formula ${ }^{3}$ :

$$
\Delta w_{l i}^{(3)}(k)=\eta \delta_{l}^{(3)} o_{i}^{(2)}(k)+\alpha \Delta w_{l i}^{(3)}(k-1)
$$

The same can be introduced hidden layer weight value formula:

$$
\Delta w_{l i}^{(2)}(k)=\eta \sigma_{i}^{(2)} o_{j}^{(1)}(k)+\alpha \Delta w_{l i}^{(2)}(k-1)
$$

Summarize the algorithm steps are as follows:

- Determine the structure of BP neural network, that is, determine the number of input layer, output layer and hidden layer node. Give the initial value and the learning efficiency of each layer's weight coefficient.

- Give the input $\operatorname{rin}(\mathrm{k})$ and output $\operatorname{yout}(\mathrm{k})$ of the system and calculate the error $E(\mathrm{k})$.

- Calculate the input and output of neurons in each layer of neural network, and the output of output layer is the three adjustable parameters of PID controller.

- Calculate the PID controller output $\mathrm{u}(\mathrm{k})$.

- Learning through neural networks, online adjustment of the weighted coefficient, to achieve adaptive control of PID parameters.

- $\quad$ Set $k=k+1$, return to the first step.

\section{Application of neural PID control system for main steam temperature system in power plant}

Thermal power plant power supply system by the boiler, steam turbine generator and other equipment. There are many factors that determine the stability and efficiency of the thermal power plant, and the main steam temperature of the boiler is the most important factor that affects the 
stability and accuracy of the power plant. And the main steam temperature control system commonly used in power plants is a PID cascade control system.

Neural network-based power plant main steam temperature control scheme, is to combine the neural network with the traditional PID controller to make up for the artificial tuning PID parameters inaccuracy. The block diagram of the main steam temperature control system of power plant controlled by neural network is shown in Fig.2.

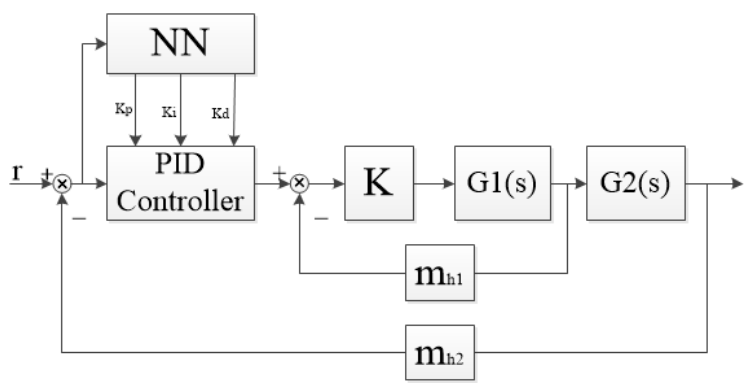

Fig.2 Power plant main steam temperature control system

The main steam temperature control system has many features. Such as the fast response, high precision, more interference ${ }^{6}$. It is usually required that the main steam temperature be controlled as accurately as possible rather than within a certain safety margin. In fact, there is a saying that about every 5 degrees lower thermal efficiency decreased by $1 \%$. Under different loads, the system has different transfer function. The formula is shown in the table1.

Table1 The formula table

\begin{tabular}{|c|c|c|c|c|}
\hline $\begin{array}{c}\text { Load } \\
\text { Value }\end{array}$ & $37 \%$ & $50 \%$ & $75 \%$ & $100 \%$ \\
\hline $\begin{array}{c}\text { Transfer } \\
\text { Function }\end{array}$ & $\frac{5.07}{(1+28 s)^{2}}$ & $\frac{3.067}{(1+25 s)^{2}}$ & $\frac{1.657}{(1+20 s)^{2}}$ & $\frac{0.815}{(1+18 s)^{2}}$ \\
\hline
\end{tabular}

\section{Simulation of main steam temperature control system based on neural network.}

\section{4-1. Simulation Analysis of Different Control Systems under the Same Load}

The classic PID control system of the $100 \%$ load value is simulated. The experimental results are shown in Fig.3.

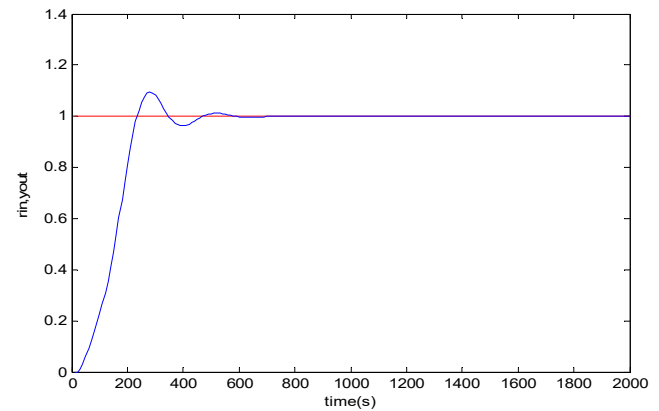

Fig.3 tracking curve based on classical PID control system

The PID control system based on BP neural network of the $100 \%$ load value is simulated, and the experimental results are shown in Fig.4 and Fig.5.

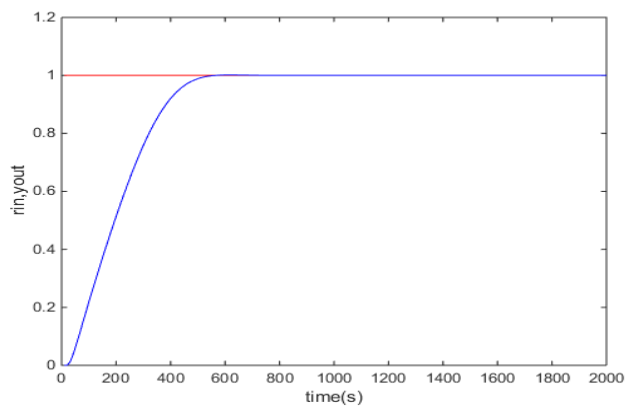

Fig.4 Tracking curve of PID based on BP neural network
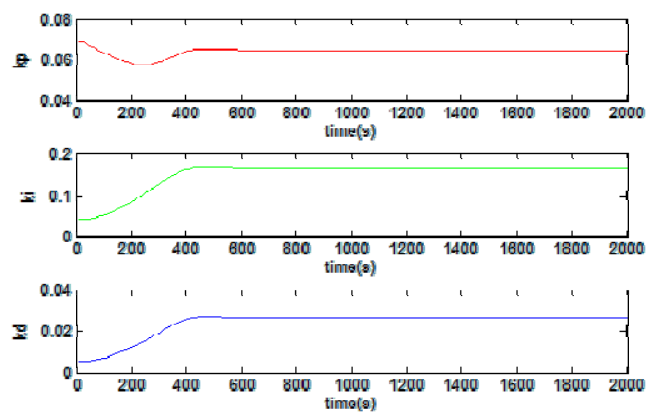

Fig. $5 k_{p}, k_{i}, k_{d}$ Parameters curve

By comparing the two tracking curve, we can get the result that the PID controller based on $\mathrm{NN}$ has good performance, small overshoot, and short adjustment time. And we can perfectly determine the three adjustable parameters of the PID to achieve the best control effect.

\section{4-2. Simulation analysis of PID control system based on $B P$ neural network under different loads}


The experimental simulation analysis of different loads under PID simulation combined with neural networks is analyzed. The experimental results are shown in Fig.6. The left figure shows the tracking curve at $37 \%$ load and the right figure shows the tracking curve at $100 \%$.
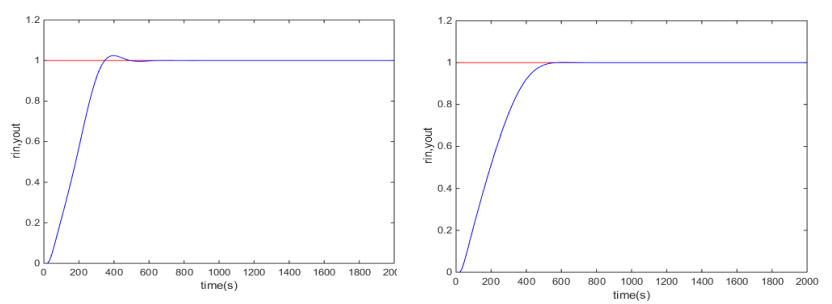

Fig.6 Tracking curve of PID based on BP neural network

Under different loads, simulation results show that the system is good. Especially when the system is running at full capacity, the best control effect can be achieved perfectly.

\section{4-3. Simulation analysis of the interference immunity of the control system}

Due to the interference of the main steam temperature control system in thermal power plants, the anti-interference ability of the system should also be improved in practical application. In the simulation can add a 0.1 interference to simulate the actual power plant interference signal. The experimental results are shown in Fig.7.

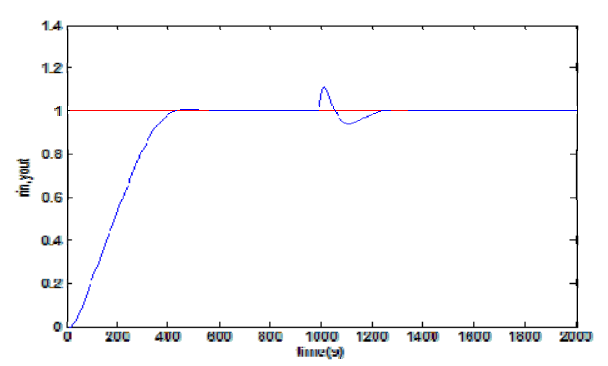

Fig.7 Step response curve plus interference

According to the simulation diagram, although there is fluctuation in the interference, the system quickly returns to its normal state. This shows that the control system has a good anti-jamming capability.

\section{Conclusion}

At present, the neural network PID control system is widely used in industry, agriculture and other fields. This control system also controls the drying of the barley malt, the raw material for beer. In addition, it can also be used in car medical thermostat control system. After we continue to explore, in the future neural network control must have a greater application prospects.

\section{Acknowledgements}

The research is partly supported by the Research Fund for the Reform in Education from Tianjin Municipal Education Commission of China (171005704B). It is also supported by the Science \& Technology Fund Planning Project of Tianjin Higher Schools (20120831, 20140710-1400020005). The design of the system is also supported by Tianjin Wiseman Optical Instrument CO., LTD.

\section{References}

1. Zhiyong Meng, Guoqing Yu, Rui Jin. Design of PID Temperature Control System Based on BP Neural Network. Applied Mechanics and Materials, 602-605: 1244-1247, 2014.

2. Zhiyuan Liu, Jianhong Lu, and Laijiu Chen, Prospects of application of intelligent PID controller in power plant thermal process control. Proceedings of the CSEE, 9(4): 351-369, 2002.

3. Han Li, Zhang Zhen-yu. The application of immune genetic algorithm in main steam temperature of PID control of BP network. Physics Procedia, 24: 80-86, 2012.

4. Wei Jiang, Xuchu Jiang. Design of an Intelligent Temperature Control System Based on the Fuzzy Self-Tuning PID. Procedia Engineering, 43:307-311, 2012.

5. S. Rominus Valsalam, Anish S, B. Raja Singh. Boiler Modelling and Optimal Control of Steam Temperature in Power Plants. IFAC Proceedings, 42(9):125-130, 2009.

6. İlhan Kocaarslan, Ertuğrul Çam. An adaptive control application in a large thermal combined power plant. Energy Conversion and Management, 48(1): 174-183, 2006. 